\title{
XVI, \\ Drei Fälle von tödtlich verlaufenen Ohrleiden, nebst Beiträgen zur pathologischen Anatomie des Gehörorganes.
}

\author{
Ton \\ Dr. K. Bürkner, \\ Göttingen.
}

In Anbetracht der Wichtigkeit einer Erweiterung unserer pathologisch-anatomischen Anschauungen im Gebiete der Ohrkrankheiten erscheinen mir nachfolgende Fälle der Veröffentlichung werth.

\section{Otitis media supparativa chronica dextra; Caries des Felsen- beins; eitrige Meningitis; Tod.}

F. E., 36 Jahre alt, Gerichtsschreiber in Grossalmerode, litt seit 11 Jahren an einem "geringftigigen Ohrenflusse ", welcher auf Anrathen eines Arztes als "der Gesundheit zuträglich" frïher nicht behandelt worden ist, zumal da sich Pat. stets beschwerdefrei gefühlt hat. Am 3. März 1881 trat plötzlich heftiges Ohrenreissen, hochgradige Schwerhörigkeit und Kopfschmerz ein und die bis dahin reichliche Eiterung verminderte sich erheblich; bald daranf verfiel Pat. zeitweise in $\mathrm{K}_{\mathrm{r}} \mathrm{am} \mathrm{m}$ fe, in lange Zeit anhaltende Delirien; am 10. März liess er den Harn unter sich gehen und wurde ganz apathisch. In der Nacht vom 11. zum 12. März wurde ich zur Consultation geholt, um eventuell eine Operation vorzunehmen.

Ich fand den Kranken in comatösem Zustande; nur auf jede Berührung des rechten Ohres reagirte er äusserst heftig; träg e $\mathrm{Pu}$ pillen, frequente Athmung, Puls 120, Temperatur (Vormittags) 390. Das linke Ohr erwies sich als normal; die rechte Ohrmuschel hart, steif, vom Schädel abstehend, ohne dass sich am Warzenfortsatz eine erhebliche Schwellung gezeigt hätte; dem Sternocleidomastoideus anf $3 \mathrm{~cm}$. nach unten folgend, erstreckte sich eine ödematöse Schwellung; in der Tiefe des Gehörgangs waren Granulationen zu erkennen, zwischen welchen die Sonde nach hinten oben etwa $1 / 2 \mathrm{Cm}$. vor dem Trommelfelle in eine cariös s Höhle glitt; das Trommelfell, soweit es zu sehen war, erschien verdickt, schwartig, von rosa Farbe. 
Da bei dem Zustande des Patienten, der den Eindruck eines Moribundus machte, an eine Operation nicht zu denken war, da ich vielmehr den letalen Ausgang für die nächsten 3-4 Tage sicher in Aussicht stellen musste, beschränkte ich mich auf Verordnung von Spir. vin. rectificatiss., wodurch die Granulationen bis zu dem am 15. März erfolgten Tode eine Abnahme erlitten.

Ueber den Sectionsbefund habe ich von dem behandelnden Arzte, Herrn Dr. Rode, leider nur wenige ungenaue Notizen erhalten können. Es wurde nur der Schảdel eröffnet. Das Schlä fentbein war uber der Paukenhöhle und dem Gehörgange schwarz, dünn, bröcklig; die Dura mater in der ganzen Umgebung des Felsenbeines injicirt und verdickt, bis zum Cerebellum mit Eite $r$ bedeckt. Sinus petrosus mit Blutgerinnseln gefullt; die Paukenhöhle enthielt schmierigbreiige Massen, von den Granulationen war gar nichts zu sehen.

Eines Commentares bedarf dieser Fall nicht. Die Symptome waren nicht zu verkennen, über die Prognose konnte kein Zweifel herrschen, die anatomischen Veränderungen entsprechen der Diagnose vollkommen. Die Krankengeschichte bietet, wie auch die folgende, ein warnendes Beispiel für jene Fälle von verschleppten Ohreiterungen, welche Jahre, Jahrzehnte lang scheinbar harmlos bestehen und schliesslich doch noch so leicht in kurzer Zeit einen so verhängnissvollen Verlauf nehmen.

\section{Otitis media suppurativa chronica bilateralis; Thrombose durch Phlebitis des Bulbus jugularis sinister; Tod.}

A. F., 20 Jahre alt, Ackersmann aus Gandersheim, litt seit 13 Jahren in Folge von Scharlach an beiderseitigem Ohrenflusse mit mässiger Schwerhörigkeit; da ihm der Ausfluss keine Beschwerden verursachte, unterliess er jede Behandlung. Am 24. September 1880 spürte er bei der Heimkelır vom Felde plötzlich einen heftigen $\mathrm{Schmerz}$ im linken $0 \mathrm{hre}$, ans welchem von dieser Zeit an kein Eiter mehr abfloss; nach kurzer Zeit stellten sich wiederholt Schuttelfröste, Erbrechen und Schwindel ein, so dass Pat. gezwungen war, im Bett zu bleiben; der zugezogene Arzt vermuthete einen Magenkatarrh und verordnete Wismuth. Die Symptome steigerten sich in den nächsten Tagen noch erheblich, und namentlich machte sich der Ohrsehmerz mehr und mehr geltend, weshalb der Kranke mir am 3. October vorgestellt wurde.

Patient fieberte beträchtlich, war durch die Schmerzen sehr geschwächt, aber bei vollem Bewusstsein; das Gehen war ihm wegen der Schwäche, mehr aber in Folge des $\mathrm{Sch}$ in dels fast unmöglich, bei jedem Schritte taumelte der Kranke. Das rechte $0 \mathrm{hr}$ eitert, das Trommelfell zeigt eine grosse centrale Perforation, durch welche die Paukenhöhlenschleimhant hochroth gefärbt 
zu sehen ist; Hörweite circa 20/150. Links: Gehörgang verengt, mit käsigen Brocken gefillt, Trommelfell blass. roth, dick, hinten unten perforirt; Ohrmuschel hart; Warzenfortsatz nicht ödematös, hingegen erstreckt sich im Verlaufe der Jugularis interna bis etwa drei Finger breit unter den Warzenfortsatz herab eine sehrempfindliche Induration, durch welche ein Thrombus ziemlich sicher zu fühlen ist. Hörfunction herabgesetzt (circa $15_{/ 150}$ ).

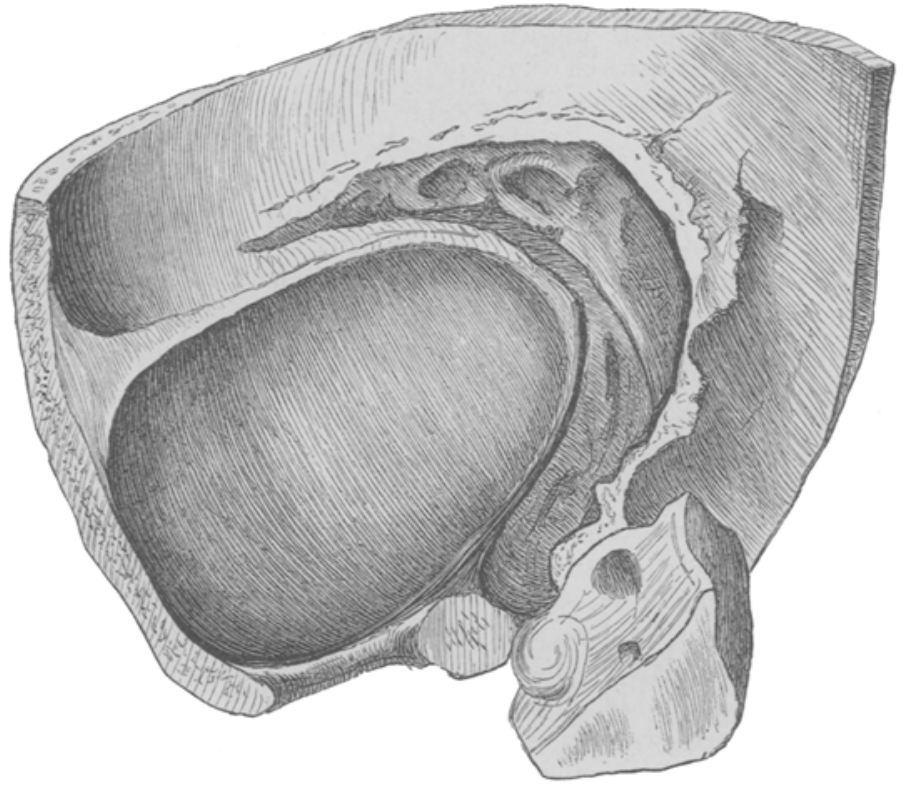

Fig. 1.

Mit Ruicksicht auf die Thrombose der Jugularis, welcher aller Wahrscheinlichkeit nach sich auch auf den Hirnsinus ausdehnen musste, konnte nur eine exspectative Therapie eingeschlagen werden: Ausspritzen der Gehörgänge mit 2 proc. Carbollösung, Umschläge auf die schmerzhafte Gegend, Bettruhe. Patient liess sich nicht abhalten, in seine Heimath zu reisen, wurde mir aber am 8. October wieder zugefiihrt.

Während in den ersten beiden Tagen nach der ersten Untersuchung die Beschwerden zuriickgegangen waren, hatte sich in den letzten 3 Tagen eine erhebliche Verschlimmerung eingestellt; die $\mathrm{S}$ ch üttelf $\mathrm{r} \ddot{o}$ ste waren immer häufiger eingetreten, das Fieber blieb auf gleicher Höhe, der Schmerz, besonders der am Nacken, hatte stetig zuge nommen; der $\mathrm{Schwindel}$ bestand nach wie vor, nur das Erbrechen war seltener geworden. Patient war apathisch, bestand aber darauf, nach Hause gebracht zu werden. Objectiv wạr 
am linken Warzenfortsatze eine Röthung und Schwellung nachweisbar, die Induration am Halse trat noch stärker hervor und war noch schmerzhafter als am 3. October; die Venen des Gesichts und die Jugularis externa waren erweitert; am linken A g e bestand leichtes Oedem. Hohes Fieber.

Am 11. October trat der am 8. mit Sicherheit prognosticirte Tod unter den Erscheinungen der Pyämie ein.

An dem mir übersandten linken Schläfenbeine fand ich folgende Veräıderungen:

Knochen dünn; Dura mater normal, Ohrmuschel normal; im 0 s t y mpanicum (vordere untere Wand des äusseren Gehörganges) eine ovale Ossificationslü cke von 4 und $3 \mathrm{Mm}$. Durchmesser, von normaler Haut iiberzogen. Trommelfell etwas dick, weiss, hinten unten runde Perforation von $3 \mathrm{Mm}$. Durchmesser mit wulstigen Rändern. Der membranöse Sinus transversus war bereits geôffnet; der Sulcus enthält vom Foramen jugulare an bis etwa 2,5 Cm. vor der Einmündung des Sinus sagitallis einen S-förmigen, festen, rothbraunen Thrombus mit unregelmässig zerklüfteter oberfläche und von einer Dicke von 0,3 bis $1,5 \mathrm{Cm}$. Die Jugularis war leider dicht an der Schädelbasis unterhalb des Bulbus abgeschnitten, doch war letzterer mit in die Thrombosirung einbezogen. Das Gerinnsel war am oberen Ende stark verjüngt, an den Biegungsstellen am dicksten. Nach $\mathrm{Ab}$ präparirung des Bulbus zeigt sich dessen der Fossa jugularis zugekehrte Wand in einer geringen Ausdehnung verfärbt, wulstig, infiltrirt. In der papierdünnen Fossa jugularis des Schläfenbeines lassen sich, genau jener Stelle am Bulbus der Vene entsprechend, rothe Punkte und feine Löcher erkennen, ohne dass Caries nachweisbar wäre; es handelt sich also um eine Dehiscenzenbildung am Boden der Paukenhöhle. Was letztere anbelangt, so war sie zum Theil mit schmierig-weissen, dicken Eitermassen angefüllt; die Gehörknöchelchen (vom Tegmen her freigelegt) sind normal, ebenso der Warzenfortsatz. Auch das häutige Labyrinth zeigte keine makroskopische Veränderung.

Dieser Fall scheint mir besonders interessant $z u$ sein wegen der erheblichen Grösse des Thrombus, den ich leider nicht in die Jugularvene weiter verfolgen konnte. Die Dehiscenzbildung war auf den Boden der Paukenhöhle und auf den Gehörgang (Os tympanicum) beschränkt, Tegmen tympani und Warzenfortsatz waren solide; zweifellos war die Phlebitis des Bulbus in Folge von Eitersenkung oder Fortleitung der Entziundung durch den dehiscenten Fundus tympani entstanden und hatte zu der secundären Thrombosirung gefuhrt. Der Tod war durch das pyämische Fieber herbeigeführt; wenigstens hatten sich nach Aussage des behandelnden Arztes, welcher anch die Obduction vorgenommen hatte, in den Organen der Brust- und Bauchböhle 
keine Metastasen oder sonstige erhebliche Affectionen vorgefunden. Auch sprach ja der Verlanf der Krankheit eher für Pyämie als für embolische Processe.

III. Otitis media suppurativa acuta (?) sin. Caries. Meningitis. Tod.

Hermann W., 17 Jahre alt, Müller aus Landolfshansen, wurde nach längerem Unwohlsein, das sich in Rücken- und Kopfschmerzen, Mattigkeit und Frostgefuhl äusserte, am 10. Januar 1882 in die medicinische Abtheilung des Ernst-August-Spitales aufgenommen, wo ich durch die Güte des Herrn Professor Ebstein Gelegenheit zu einer genauen und fortgesetzten Beobachtung hatte. Pat. gab an, seit $\checkmark$ Tagen Ausfluss aus dem linken 0 hre und seit 3 Tagen eine linksseitige Gesichtslähmung zu haben; letztere ist sehr auffallend; die Oberlippe ist geschwollen, nur rechts beweglich; auch die linke Unterlippe und Stirnhälfte sind gelähmt; Uvula steht etwas nach rechts. Ausserdem besteht Lungenkatarrh (besonders links hinten), das Sputum ist eitrig-sehleimig, gelbgrïnlich gefärbt. Puls 80, Resp. 20, Temp. 39,2.

Die Diagnose wurde auf Meningitis (tubereulosa?) gestellt.

Am 11. Januar sah ich den Kranken zuerst. Am rechten Ohre findet sich ausser drei kleinen A uricularanhängen vor dem Tragus nichts Abnormes; der linke Gehörgang ist in der Tiefe roth, geschwollen, vor dem Trommelfell, welches im hinteren unteren Quadranten eine Perforation zeigt, liegen kleine Granulationen; die Gegend am Hammergriff ist verdickt und weiss, so dass das Manubrium nicht dentlich erkennbar ist; am unteren Rande des Trommelfells pulsirender Reflex. Die Eiterung scheint nicht sehr copiös, das Secret ist von grünlicher Farbe und scharfem, käsigem Geruch. Warzenfortsatz durchaus nicht druckempfindlich. Gehör 10/120.

Therapie: Ansspritzungen, Insufflationen von pulverisirt. Jodoform und Borsäure ana.

12. Januar. Vor den Granulationen bildet sich eine ödematös e Stelle an der hinteren oberen Wand des knöchernen Gehörgangs, welche in den nächsten 3 Tagen allmählich wieder abschwillt.

20. Januar. Da die Granulationen gewachsen sind, werden die grössten derselben, zwei linsengrosse Tumoren, mit der Wilde'schen Schlinge abgetragen; darauf Lapisätzungen, in den nächsten 6 Tagen fortgesetzt, bis die Granulationen verschwunden sind und die jetzt grösser erscheinende Perforation freiliegt. Gehör 12/120.

23. Januar. Kopfschmerz geringer, Nacken etwas steif, Neigung zu Collaps, kahnförmig eingezogenes Abdomen.

5. Februar. Fast sämmtliche klinischen Symptome verschwunden; kaum geringer Spitzenkatarrh, gutes Allgemeinbefinden, Facialis frei. Die Ohreiterung ist indessen profus.

Diese relative Euphorie halt sich den Februar hindurch; auch das Ohr zeigt Neigung zur Besserung: der A usfluss nimmt unter regelmässiger Anwendung vou Borsänre ab, es besteht 
durchaus keine Empfindlichkeit, das Gehör schwankt zwischen 10/120 und 18/120. Nur in den letaten Tagen des Februar besteht eine geringe Schwellung vor dem Tragus, die jedoch am 1. März verschwunden ist.

6. März. Die Schmerzen im linken Ohre treten plötzlich mit grosser Heftigkeit wieder auf, zugleich lässt die Eiterung fast ganz nach; die bis dahin normale Temperatur steigt voribergehend auf 38,5 . Uhr ad conch. undeutlich.

11. März. Facialis wieder paralytisch, Ohrschmer$z$ en seh $r$ in te n si $v$, ohne objective Veränderung, mit Ausnahme einer gleichmässigen Röthung am Trommelfell. Gehör hat merklich abgenommen. Uhr wird nicht mehr gehört, stimmgabel besser rechts.

20. März. Wieder reichlichere Otorrhoe. Trommelfell unverändert; linke Pupille enger.

25. März, Schnell oscillirender Nystagmus beiderseits (anch ohne Fixation). Hörfunction aufgehoben.

26. März. Linker A bducens vollständig gelähmt. Eiterung profus; Perforationsränder etwas wulstig; Paukenschleimhaut hochroth. Somnolenz.

29. März u. f. Schwäche im rechten Beine (Parese), keine Reflexe durch Nadelstiche zu erzielen; links Reflexerregbarkeit; Bauch eingesunken, Stuhl retardirt. Conjunctivitis, Schmerzen in allen Trigeminusästen. Nystagmus nur noch bei Fixation. Erbrechen, Somnolenz. Ohrbefund unverändert.

4. April. Tod unter unregelmässig werdender Respiration.

Sectionsbefund. (Auszug aus dem Sectionsprotocolle des Herrn Prof. Orth, aufgenommen cirea 30 h. p. m.)

An der ganzen Oberfläche der inneren Schädelwand weissliche Osteophyten. Dura durchsichtig, gespannt; Pia mattglänzend, Hirnwindungen deutlich abgeplattet; durch die Pia stark gefillte Venen erkennbar. Vorderes Ende des linken Kleinhirnlappens mit dem hinteren Rand des Felsenbeins, dicht hinter der Durchtrittsstelle des Trigeminus, und um den Meatus audit. intern. herum verwachsen. Am Bulbus olfactorius starke Gefässinjeetion; um das Chiasma herum starke Triibung und eitrige Infiltration. Pons linkerseits abgeplattet, etwas in die Länge gezogen. An der Stelle, wo die Verwachsung: war, sieht man eine sulzig-graue Masse mit eingesprengten gelben Flecken, welche sich in die Pia unter dem Trigeminus her nach dem Pons and zwischen Pons und Cerebellum nach der Medulla oblong. hinerstreckt. Der linke Abducens etwas platter als der rechte und in seinen inneren Partien etwas grau; auch am Trigeminus ist die Färbung links weniger weiss als rechts. Facialis und Acusticus der linken Seite sind vollständig in die sulzige Masse aufgegangen und als weisse Stränge nicht mehr nachweisbar. Auf einem Schnitt durch die Adhäsionsstelle zeigt sich im Cerebellum ein kirschkerngrosser Knoten, aus sulzig-graner Masse mit gelben Flecken bestehend. Bei einem Einschnitt in den Pons, dicht unter dem Austritt des Trigeminus wird ein kirschgrosser Abscess eröffnet, aus welchem ein gelber Eiter sich ergiesst. Von der Oberfläche des Pons ist die Abscess- 
wand $12 \mathrm{Mm}$. entfernt. Die Abscesshöhle besteht aus einer sulziggrauen, besonders an der einen Seite reichlich vascularisirten Masse, welche an der äusseren Seite die Dicke von 2, an der inneren von $4 \mathrm{Mm}$. zeigt, aber nicht seharf umsehrieben ist, sondern sich nach aussen in die angrenzende Hirnmasse verliert. Die Abscesswand wölbt sich stark in den IV. Ventrikel vor, nimmt die ganze linke Hälfte desselben ein und reicht etwa $5 \mathrm{Mm}$. über die Mittellinie auch nach rechts hiniber. Ein Schnitt $3 \mathrm{Mm}$. von dem hinteren Ende des Pons trifft fast die hintere Wand des Abscesses. In der Medulla oblong. ist vom Abscess nichts zu sehen, hingegen erscheint der Centralkanal von einer anffallig weichen Masse umgeben, welche ungefähr $1 \mathrm{Mm}$. im Durchmesser haltende Flocken darstellt. Dicht vor dem Trigeminus trifft der Sehnitt eine Erweiterung des Ventrikels, wie sie auch an den Grosshirnventrikeln nachweisbar ist. Striae acusticae sind links durch den sich wölbenden Abscess weit auseinander gedrängt und sehen etwas grauer aus als rechts. An der Dura in der. Umgebung des Meatns vascularisirte Haut.

An der First des Felsenbeins drei cariöse Stellen von $2 \mathrm{Mm}$. Durchmesser; dicht über dem Sulcus sigmoid. ist der Knochen von weichen, ein wenig prominirenden, theils schmierigen, theils atlasglänzenden, gelblichweissen Massendurchsetzt, welche sich in einer unregelmässig buchtigen mit glänzender Wandung ausgekleideten Höhle befinden, die die ganze hintere Abtheilung des Felsenbeins einnimmt und an den oben bezeichneten drei Stellen mit der Schädelhöhle, nach oben und aussen mit dem Vorhofe in Verbindung steht. Die Paukenhöhle ist gegen den Gehörgang zum grössten Theil durch das Trommelfell abgesehlossen, communicirt aber durch ein einige Millimeter grosses Loch mit demselben. Von Gehörknöchelchen ist nichts mehr zu finden. Mit dem Nerven setzt sich die erwähntesulzig-graue Masse in das innere Ohr fort, von dessen Bestand theilen ebenfalls nichts Besonderesmehr erkannt werden kann, da ihre Stelle von der grauen Masse und etwas Eiter eingenommen ist.

In diesem Falle muss die Zerstörung des Gehörapparates sehr rasch vor sich gegangen sein; zu Weihnachten erkrankte Patient angeblich zuerst und Anfang A pril findet sich das Felsenbein zur Hälfte zerstört, das Labyrinth in eine grosse cariöse Höhle einbezogen, der Schallleitungsapparat verschwunden. Selbst, wenn man, entgegen der Anamnese, annehmen wollte, - and diese Annahme wäre wohl berechtigt - dass ein cariöser Process im Schläfenbeine schon längere Zeit schleichend bestanden habe, so beweisen doch die sorgfältig vorgenommenen Hörprüfungen, dass die Störung zur Zeit der Aufnahme des Patienten noch relativ gering war, da die Hörfunction erst in den letzten Wochen sehr geschwächt und schliesslich aufgehoben, im Anfang ziemlich gut 
war. Auffallend war die ganz wesentliche Besserung, welche sich in sämmtlichen Symptomen zu Ende Januar, und zwar nach der Entfernung grösserer Granulationen, einstellte; während bis zum Tage der Operation die meningealen Symptome sehr in den Vordergrund traten, verloren sich sämmtliche Hirnerscheinungen nach dem localen Eingriff, selbst die Facialislähmung liess nach. Dieser Umstand erklärt sich wohl durch die nach der Operation leichter und gründlicher $\mathrm{zu}$ bewerkstelligende Reinigung des Ohres, und es hatte in der That den Anschein, als ob nun auch die Mittelohrentzündung in der Heilung begriffen sei, obwohl doch vielmehr der verborgene Process in der Tiefe immer mehr und mehr um sich gegriffen haben muss, Der rasche Zerfall, die weitere Ausdehnung der Caries datirt offenbar erst vom 6. März, an welchem Tage plötzlich zugleich mit einer Eiterretention wieder schwere Hirnsymptome eintraten.

Obwobl ich von Anfang an und auch später, nach Eintritt der Verschlimmerung überzeugt war, es müsse sich um Caries des Schläfenbeins handeln, gelang es mir bei wiederholten, sorgfältigen Sondirungen niemals, eine cariöse Stelle aufzufinden, ein Umstand, der sich aus der, auch bei der Section erwiesenen normalen Beschaffenheit des Gehörganges und der Paukenhöhlenwandungen erklärt. Hätte sich das Bestehen einer cariösen Knochenerkrankung sicherstellen lassen, so wäre in dem Stadium der Besserung unbedingt die chirurgische Eröffnung des Warzenfortsatzes indicirt gewesen; die Verschlimmerung trat ja dann leider so rasch und intensiv ein, dass jede Operation zu spät gekommen und durch den Zustand des Patienten direct contraindicirt gewesen wäre.

IV. Nicht uninteressant scheint mir ferner folgender Fall von nervöser Taubheit in Folge eines vom IV. Ventrikel ansgehenden Hirnsark orms zu sein, welchen ich mit freundlicher Erlaubniss des Herrn Prof. Ebstein, auf dessen Abtheilung ich den Kranken zu untersuchen Gelegenheit hatte, an dieser Stelle veröffentliche.

Heinrich H., 36jähriger Tagelöhner aus Rössing, wurde am 30. Januar 1882 ins Ernst-August-Hospital aufgenommen, da er an zunehmender Schwäche in den Beinen, häufigem Schwindel und Kopfschmerz leidet und seit dem Juli 1881 blind ist.

Aus dem Status praesens entnehme ich Folgendes:

Der kräftig gebaute und wohlgenährte Patient vermag nicht zu gehen und nur schlecht zu sitzen, klagt uber Kopfschmerz, Schwindel, rechtsseitige Taubheit. Es wird nur ein geringer Lichtschein percipirt, 
beiderseits besteht Neuroretinitis (Stauungspapillen, beginnende Atrophie); Pupillen maximal weit, ganz reactionslos; Bewegungen beider Angen, besonders des rechten, sehr beschränkt, rechter Abducens gelähmt, Internus paretisch, Inferior und Superior reagiren nicht normal; links Internus paretisch. Bei seitlicher Fixation, sowie beim Blick nach oben und unten, Nystagmus. Trigeminus rechts etwas paretisch; leise Berdhrungen der Gesichtshaut werden rechts nicht gespiurt, Nadelstiche und stärkere Berthrungen undeutlicher, als links. Bei leiser Beruhrung der rechten Cornea erfolgt kein Lidschluss, auch fehlen von der rechten Nasenschleimhaut aus Reflexbewegungen. Fa cialis rechts ebenfalls paretisch; der willkürliche Lidschluss sehr matt, Frontalis, Lippen- und Mundäste paretisch. Besonders auffallend treten die Lähmungserscheinungen beim Lachen und Sprechen hervor; auch die rechte Gaumenhälfte bewegt sich weniger ausgiebig als die linke. Acusticus erscheint, bei vollständig negativem $\mathrm{Be}-$ funde am rechten Ohre und absoluter rechtsseitiger Taubheit gleichfalls gelähmt. Ein geringer Grad von Arhythmie und Herzklopfen deuten auf Störungen im Gebiete des Vagus; eine auffallende Schwerbeweglichkeit des Kopfes auf Parese des Accessorius; die Zunge weicht beim Ausstrecken nach rechts, beim Zurückziehen nach links; die Sprache ist abnorm verlangsamt, scandirend, monoton (H y poglossusparese). Auch die Geschmacksempfindungen sind abnorm: Zuckerlösung gibt rechts salzige, links bittere Empfindung.

Sensibilitätsstörungen bestehen weder am Rumpfe, noch an den Extremitäten; die Sehnenreflexe sind erhalten; beide Körperhälften sind paretisch, die rechte mehr als die linke; die Bewegungen sind sehr unsicher.

Bei der in den letzten Tagen des Januar zum ersten Male und später wiederholt vorgenommenen Untersuchung der Ohren erweisen sich beide Trommelfelle als normal, am rechten zeigt sich eine unbedentende diffuse Trübung; rechts besteht vollständige Taubheit, links annähernd normales Gehör. Die Haut des rechten Gehörganges ist weniger sensibel, als die des linken. Auszug aus dem am 23. Mai 1882 aufgenommenen Sectionsprotocoll (Prof. Orth).

Kräftiger Mann, Schädel leicht abhebbar, Dura weniger durchscheinend als normal, stark gespannt, Gefässe bluthaltig; Pia mattglänzend, der Dura fest anliegend. Bei Herausnahme des Gehirns zeigt sich rechts am Pons ein Tumor, welcher der vorderen Hälfte der hinteren Felsenbeinwand fest anliegt, von der Dura leicht zu trennen ist, dagegen mit dem Facialis und Acusticas, welche in dem Tumor zuverlaufen scheinen, in den Meatus audit. int. sich hineinzieht. An seinem äusserlich sichtbaren Theile hat der Tumor eine Länge und Breite von circa 4 Cm.; er verjüngt sich nach vorn zu ein wenig; sein vorderes Ende entspricht der vorderen Grenze des Pons, wahrend er nach hinten zu die Ponsgrenze um circa $1 \frac{1}{2} \mathrm{Cm}$. uberragt. Die rechte Seite des Pons ist fast gänzlich zerstört, so dass direct neben der normal verlanfenden Arteria basilaris bei der Lagerung des Hirns auf der convexen Seite der Pons ganz steil abfält; an der so gebildeten First liegt der rechte 
Abducens dicht der Basilararterie an, in der Tiefe läuft zwischen Tumor und Pons der plattgedrückte Trigeminus nach vorn und tritt dicht neben dem Oculomotorius hervor. Der vordere Abschnitt der Medulla oblong. ist nach vorn za verschmälert; das Infundibulum sehr erweitert, Höhle des Türkensattels entsprechend weit und tief, Zirbeldrüse weich, röthlich, mit kleiner Cyste, in der Tiefe zeigt sich die Keilbeinhöhle eröffnet. Starkes Oedem des Bindegewebes in der Gegend des Chiasma; die Optici sehen auf dem Durchschnitt dick und

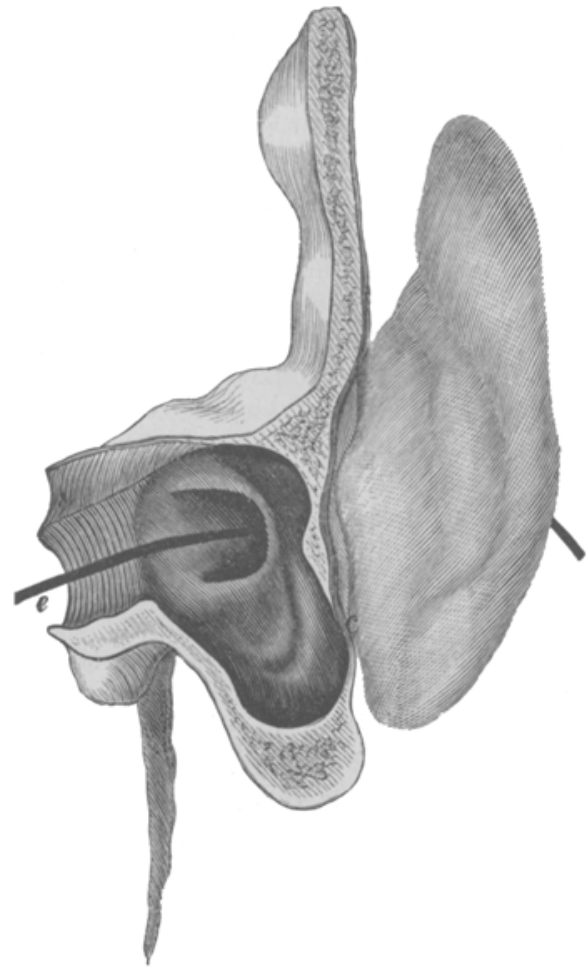

Fig. 2. c. Fistelöffnung; $e$ Sonde durch don äusseren Gehörgang gefürt. wie sulzig aus, haben auch nicht die normale weisse Farbe; rechter Facialis und Acusticus sind ganz grau. Der Meat. a udit. int. erscheint erweitert. Der Tumor hat eine unregelmässig höckerige Oberfläche und elastische Consistenz, graugelbliche Farbe, ist im Centrum gallertig, Schleimgewebe ähnlich, nach der Peripherie weiss, mit kleinen braunen Partien und vereinzelten rothen hämorrhagischen Stellen. Aus'der Tiefe des Längsdurchschnittes sieht man weisse Streifen nach der Oberfläche hinziehen, die an Nervenfasern erinnern. An einem mitten durch den Pons geführten Schnitt sieht man, dass es sich weniger um Atrophie, als um Verschiebung der Bestandtheile handelt. Die Grosshirnventrikel sind erweitert, in allen Abschnitten zeigt sich das Ependym verdickt, die Hemisphären sind

durchfeuchtet. Bei der Abtragung der rechten Hemisphäre zeigt sich in der Nähe des hinteren Endes des Streifenhügels ein etwa haselnussgrosser, mit feinen, punktförmigen Hämorrhagien durchsetzter Herd in der weissen Substanz, der bis dicht an die Windungen des Schläfenlappens reicht. Nach der Eröffinung des IV. Ventrikels von oben sieht man den Tumor sich von rechts her halbkugelig vorwölben. In dem aufgemeisselten inneren Gehörgange lässt sich die Geschwulst, welche den verschwundenen Acusticus vollständig ersetzt, bis zur Macula cribrosa verfolgen; sie enthält hier nicht die geringsten Reste von Nervenfasern. Die Schnecke ist normal. Die Ge- 
schwulst erweist sich bei der mikroskopischen Untersuchung als $R$ und. z ellensarkom.

Es hat sich mithin in diesem Falle um eine rein nervöse Taubheit bei intactem Gehörorgan gehandelt; der Acusticus war vollständig in die Sarkommasse aufgegangen.

Im Anschluss an diese Fälle aus der Praxis möge hier nun noch ein Präparat meiner Sammlung beschrieben werden, das einem Schädel entstammt, welchen ich der Güte des Herrn Dr. von Brunn verdanke.

Kopf eines alten Mannes; Caries des Schläfenbeins mit Fistelöffnung nach aussen.

Am rechten Warzenfortsatze, $4 \mathrm{Mm}$. hinter dem Porus acust. extern. und in gleicher Höhe wie dieser, findet sich eine ovale 0 ef f $\mathrm{n}$ u $\mathrm{g}$ von $6 \mathrm{Mm}$. Höhe und $4 \mathrm{Mm}$. Breite, durch welehe die Sonde $3 \mathrm{Cm}$. tief nach innen dringt. Nach Herausnahme des Schläfenbeins und Durchsägung des Knochens in der Längsaxe der Fistelöffunng dureh den Warzenfortsatz, parallel mit der Anheftungslinie der Ohrmuschel, zeigt sich eine grosse Höhle, weleheden ganzen hinteren Theil des Warzentheiles und Felsenbeintheiles ein. nimmt und nach oben, aussen und innen duroh blasig aufgetriebenen, dif $n n e n$ und $m$ orseben Knochen begrenzt, am Warzenfortsatzesich in der durchsägten Fistel direct nach aussen offnet.

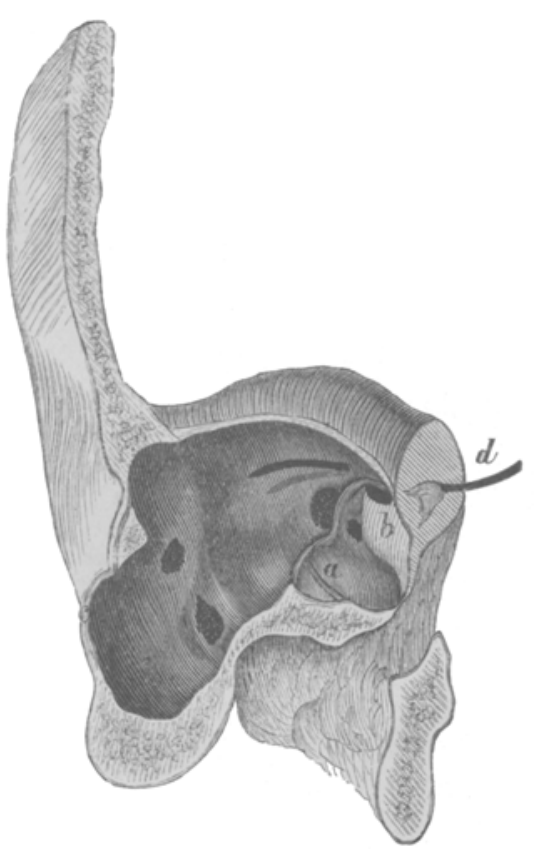

Fig. 3. a. Sulcus membr. tymp.; b. Promontorium c. Fistelöffung; $d$. Sonde durch den Canalis Faloppiae geführt.

Der Sinus transversus communicirt durch zwei circa $3 \mathrm{Mm}$. lange rundliche Löcher von möglicherweise postmortalem Ursprunge mit der Höhle; der membranöse Sinus ist indessen intact. Eine weitere Verbindung des Hohlraumes besteht mit der Paukenhöhle durch deren hintere Wand, welche fast vollständig fehlt. Auch der Facialkanal ist fast ganz in der. Höhle aufgegangen; er besteht, wie die durchgeführte Sonde ergibt, nur noch vom inneren Gehörgange aus über dem ovalen Fenster auf eine Strecke von $5 \mathrm{Mm}$. 
256 XVI. BÜRKNER, Dreí Fälle von tödtlich verlaufenen Ohrleiden.

und über dem Foramen stylo-mastoideum in der Ausdehnung von 2-3 Mm.

Von der hinteren Wand des knöchernen Gehörganges ist nur ein Drittel vorhanden; die Höhle setzt sich hier direct in den äusseren Ohrkanal fort; das Trommelfell und die Gehörknöchelchen fehlen gänzlich, der Sulcus tympanicus ist binten und unten unterbrochen, so dass er nur noch am vorderen und theilweise am unteren Ende des Gehörganges nachweisbar ist. Die innere, obere und vordere Paukenhöhlenwand sind normal, das 'Tegmen allerdings papierdünn. Das Labyrinth offnet sich nicht in die Höhle. Letztere, sowie die Paukenhöhle ist mit cholesteatomatösen Massen angefüllt und mit einer dunkelbraunen, stark glänzenden Membran austapezirt.

Ueber die Todesursache war leider nachträglich nichts mehr zu ermitteln; die Leiche war auf die Anatomie abgeliefert wor* den. Bei dem oben geschilderten Befunde ist die Möglichkeit, dass die Ohrkrankheit letal verlaufen ist, jedenfalls nicht ausgeschlossen. 